\title{
Densest Packing of Translates of the Union of Two Circles
}

\section{Fejes Tóth}

Mathematical Institute of the Hungarian Academy of Sciences, Reáltanoda 13-15, 1053 Budapest, Hungary

\begin{abstract}
Let $u$ be the union of two unit circles whose centers have a distance at most 2. Motivated by more general problems it is proved that the density of a packing of translates of $u$ never exceeds the density of the densest lattice-packing.
\end{abstract}

In the Euclidean plane let $w$ be a domain. Let $d(w)$ be the density of the densest packing of translates of $w$. Let $\bar{d}(w)$ be the density of the densest lattice-packing of translates of $w$. It is known [1], [2], [3] that if $w$ is convex then

$$
d(w)=\bar{d}(w) .
$$

An interesting field of research arises by trying to extend (1) to more general domains [4]. From some wider families of domains, which presumably share property (1) with the convex domains, we emphasize

Conjecture 1. Let $u$ be the union of two convex domains having a point in common. Then $d(u)=\bar{d}(u)$.

As a modest step in this direction we shall prove the following:

Theorem. Let $u$ be the union of two unit circles centered at a distance at most 2 from one another. Then $d(u)=\vec{d}(u)$.

Let $u_{1}$ and $u_{2}$ be translates of $u$ such that $u$ and $u_{1}$ have three points of contact, and $u_{2}$ has one point of contact with both $u$ and $u_{1}$. The densest lattice-packing of translates of $u$ is generated by the translations $u \rightarrow u_{1}$ and $u \rightarrow u_{2}$. Let $2 t$ be the distance of the centers of the two unit circles contained in $u$. Then $\bar{d}(u)$ is given in terms of $t$ by the function

$$
f(t)=\frac{2\left(\arcsin t+t \sqrt{1-t^{2}}\right)+\pi}{2 t \sqrt{4-t^{2}}+\sqrt{12}}
$$


For $0<t<1$ we have $f(t)>f(0)=f(1)=\pi / \sqrt{12}$. The maximum of $f(t)$ is attained at a value $t_{0} \approx 0.582$. Since translates of the union of two disjoint circles cannot be packed with a density greater than $\pi / \sqrt{12}$ the theorem implies that the density of a packing of translates of the union of two equal circles is at most $f\left(t_{0}\right)=0.936599 \ldots$

What can be said about the supremum of $d(u)$ extended over the set $S_{n}$ of all unions $u$ of $n$ unit circles? We shall return to this question after the proof of the theorem.

The proof of the theorem is based on an idea used in a proof of Thue's well-known theorem which claims that for a circle $c$ we have $d(c)=\vec{d}(c)=\pi / \sqrt{12}$. First we will reproduce this proof [5] in a slightly modified form. We shall denote the area of a domain $w$ by $|w|$. By a circle we shall mean an open circular disc.

Let the unit circles $c_{1}, c_{2}, \ldots$ with centers $C_{1}, C_{2}, \ldots$ form a packing. Let $z_{i}$ be the Dirichlet cell of $c_{i}$ defined as the set of those points which are nearer to $C_{i}$ than to the center of any other circle. We shall show that the density $\left|c_{i}\right| /\left|z_{i}\right|$ of any circle $c_{i}$ in its Dirichlet cell is at most $\pi / \sqrt{12}$. Writing $c_{i}=c$ and $z_{i}=z$, this means that $|z| \geq|h|$ where $h$ is a regular hexagon circumscribed about $c$.

We shall prove a sharper inequality: If $k$ is the circumcircle of $h$ then

$$
|z \cap k| \geq|h| \text {. }
$$

Let $s_{1}, \ldots, s_{n}$ be the segments cut off from $k$ by the sides of $z$. Let $F_{1}, \ldots, F_{n}$ be the orthogonal projections of $C$ to the respective sides of $z$. Since the distance between any two of the $C_{i}$ 's is at least 2 , the distance between the $F_{i}$ 's is at least 1. It follows that the $s_{i}$ 's do not overlap. Therefore

$$
|z \cap k|=|k|-\left|s_{1}\right|-\cdots-\left|s_{n}\right| \geq|k|-n|s|,
$$

where $s$ is a segment cut off from $k$ by a tangent of $c$. For $n \leq 6$ this implies the desired inequality

$$
|z \cap k| \geq|k|-6|s|=|h| \text {. }
$$

The case when $n=7$ can be settled by a very rough estimate which automatically rules out the possibility that $n>7$. Since the length

$$
\frac{4}{\sqrt{3}} \sin \frac{\pi}{7}=1.00201 \cdots
$$

of a side of a regular heptagon inscribed into $k$ is close to 1 , it is clear that the points $F_{1}, \ldots, F_{7}$ must lie close to the boundary bd $k$ of $k$, suggesting that the $s_{i}$ 's are very small. Though this is sufficiently convincing, we present the details.

The distance $C F_{7}$ attains its minimum $m=1.1383 \cdots$ if $C F_{1}=\cdots=C F_{6}=$ $2 / \sqrt{3}=1.1547 \ldots$, and all sides of the heptagon $F_{1} \cdots F_{7}$ are equal to 1 . It follows that

$$
\left|s_{i}\right| \leq \frac{1}{2}\left(\frac{2}{\sqrt{3}}\right)^{2}(2 \omega-\sin 2 \omega)=0.00424 \cdots, \quad i=1, \ldots, 7
$$


where $\omega=\arccos \sqrt{3} m / w$. Hence

$$
\left|s_{1}\right|+\cdots+\left|s_{7}\right|<0.0297<6|s|=0.724 \ldots
$$

Turning to the proof of the theorem, we consider a rectangular coordinate system $(x, y)$ in which the centers of the two unit circles contained in $u$ coincide with $(-t, 0)$ and $(t, 0)$. Let $c^{-}$and $c^{+}$be the left- and right-sided circles, and $C^{-}$ and $C^{+}$their centers. Let the translates $u_{1}, u_{2}, \ldots$ of $u$ form a packing. Let $c_{1}^{-}$, $c_{1}^{+}, c_{2}^{-}, c_{2}^{+}, \ldots$ be the set of pertaining circles. In this set let $z_{i}^{-}$and $z_{i}^{+}$be the Dirichlet cells of $c_{i}^{-}$and $c_{i}^{+}$. We define the Dirichlet cell of $u_{i}$ by $z_{i}=z_{i}^{-} \cup z_{i}^{+}$. In order to simplify the notations we suppose that $u$ is an arbitrary member of the packing, and denote its Dirichlet cell by $z$.

We shall prove the theorem by showing that

$$
|u| /|z| \leq \bar{d}(u) \text {. }
$$

We shall prove a sharper inequality: If $k^{-}$and $k^{+}$are circles concentric with $c^{-}$ and $c^{+}$of radius $2 / \sqrt{3}$ and $k=k^{-} \cup k^{+}$then

$$
|z \cap k| \geq|u| / \bar{d}(u)=|\bar{z}|
$$

where $\bar{z}$ is a Dirichlet cell in the densest lattice-packing.

Let $u_{1}, \ldots, u_{n}$ be those domains other than $u$ whose Dirichlet cells intersect $k$. Let the respective intersections be $s_{1}, \ldots, s_{n}$. Since the Dirichlet cells do not overlap neither do the $s_{i}$ 's. Therefore

$$
|z \cap k|=|k|-\left|s_{1}\right|-\cdots-\left|s_{n}\right|
$$

We claim that among $s_{1}, \ldots, s_{n}$ there are at most two whose areas are greater than $|s|$. We shall prove this by showing that, with the exception of at most two, the sets $s_{1}, \ldots, s_{n}$ are circular segments.

Let $u_{0}$ be a translate of $u$ not necessarily disjoint of $u$. Let $C_{0}^{-}$and $C_{0}^{+}$be the corresponding translates of $C^{-}$and $C^{+}$. In the arrangement of translates of $u$, consisting only of $u$ and $u_{0}$, let $z_{0}$ be the Dirichlet cell of $u_{0}$. We say that the intersection $s_{0}=k \cap z_{0}$ is exceptional if it is not empty and not a circular segment. Let $p$ be the set of points defined by the following property: If either $C^{-} \in p$ or $C^{+} \in p$ then $s_{0}$ is exceptional.

Let $a$ and $b$ be circles of radius $2 / \sqrt{3}$ centered at the points of intersection of bd $k^{-}$and bd $k^{+}$(Fig. 1). The difference $\left(2 k^{-} \cap 2 k^{+}\right) \backslash(a \cup b)$ consists of four arc-sided triangles. Let $v$ and $w$ be the triangles bisected by the $y$-axis. We claim that $p=a \cup b \cup v \cup w$. This can easily be seen by the following alternative definition of $p$ : The set $p$ consists of the centers of those circles of radius $2 / \sqrt{3}$ whose boundaries intersect both arcs constituting bd $k$.

Presently we are only interested in the part of $p$ which lies in the ring $r$ defined by $r=\left(2 k^{-} \cup 2 k^{+}\right) \backslash\left(2 c^{-} \cup 2 c^{+}\right)$. This part consists of two disjoint components whose diameter is less than or equal to 2 with equality only in the limiting case as $t \rightarrow 0$. Since the distance between centers $C_{i}^{-}$or $C_{i}^{+}$belonging to different $u_{i}$ 's is at least 2 , the assertion is proved. 


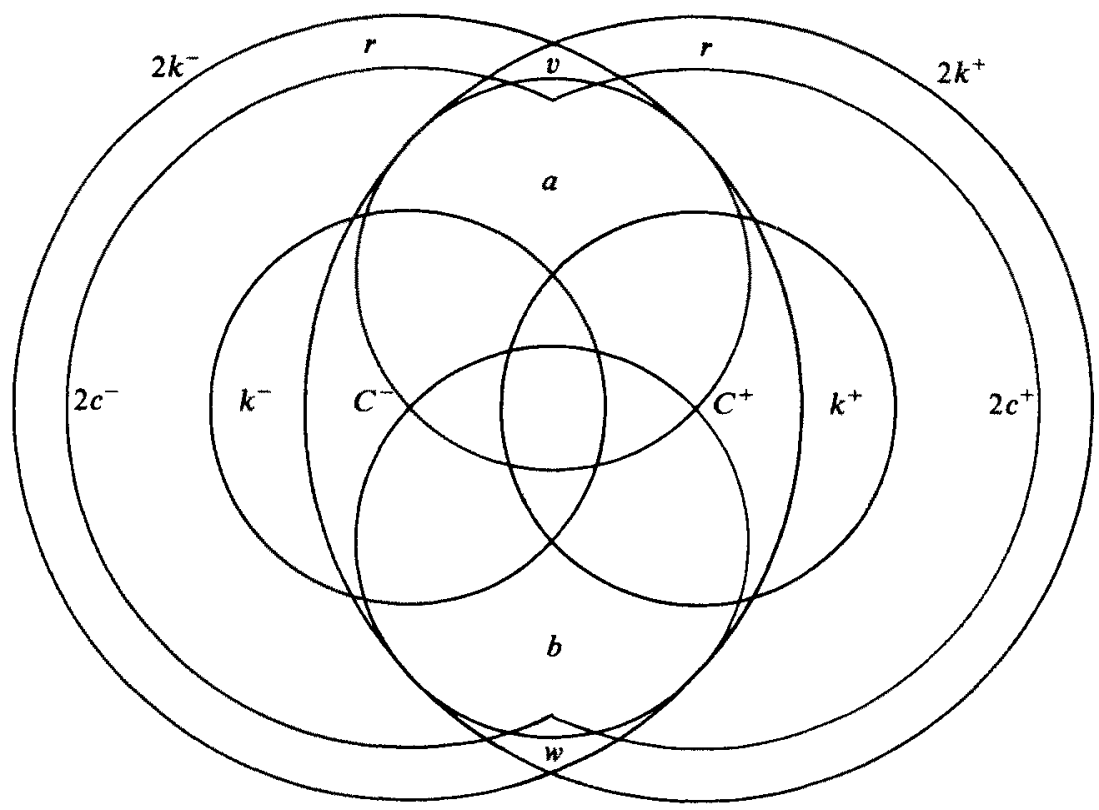

Fig. 1

Now we make use of the easily seen fact that under the sole condition that $u$ and $u_{0}$ are disjoint $\left|s_{0}\right|$ attains its maximum if $u_{0}$ and $u$ have three points of contact. Denoting $s_{0}$ in this position by $\hat{s}$, and assuming that $n \leq 6$, we have, by (3), in accordance with (2),

$$
|z \cap k| \geq|k|-4|s|-2|\hat{s}|=|\bar{z}| \text {. }
$$

Now we consider seven pairs of centers $\left(C_{1}^{-}, C_{1}^{+}\right), \ldots,\left(C_{7}^{-}, C_{7}^{+}\right)$such that of each pair at least one center is in $r$. Let $\left(C_{i}^{-}, C_{i}^{+}\right)$be a pair of centers such that $C_{i}^{-}$lies in the half-plane $x \leq 0$ and $C_{i}^{+}$lies in the half-plane $x \geq 0$. We assume that among the seven pairs of centers there are (i) two such pairs, or (ii) one such pair, or (iii) none.

Case (i). Let $C_{7}^{+}, C_{1}^{-}, C_{2}^{-}, C_{3}^{+}$be in $x \geq 0$, and let $C_{3}^{-}, C_{4}^{+}, C_{5}^{+}, C_{6}^{+}, C_{7}^{-}$be in $x \leq 0$. Translating $C_{4}^{+}, C_{5}^{+}$, and $C_{6}^{+}$through the vector $\mathrm{C}^{-} \mathrm{C}^{+}$we obtain, along with $C_{7}^{+}, C_{1}^{-}, C_{2}^{-}$, and $C_{3}^{+}$seven points in the ring $2 k^{+} \backslash 2 c^{+}$with a distance at least 2 from one another. Referring to the above proof of Thue's theorem we see that these points must be close to bd $2 k^{+}$. Therefore the original points must be close to bd $\left(2 k^{-} \cup 2 k^{+}\right)$so that $|z \cap k|$ is, by far, greater than $|\bar{z}|$.

Case (ii). We can translate the domains $u_{1}, \ldots, u_{7}$ continuously "outwards" without overlapping each other until, of each pair of centers, one center lies on bd $\left(2 k^{-} \cup 2 k^{+}\right)$, and the other lies either on bd $\left(2 k^{-} \cup 2 k^{+}\right)$or outside $2 k^{-} \cup 2 k^{+}$. We number the centers so that $C_{1}^{-}, C_{2}^{-}, C_{3}^{-}, C_{4}^{+}, C_{5}^{+}, C_{6}^{+}, C_{7}^{-}$, and $C_{7}^{+}$lie, in 


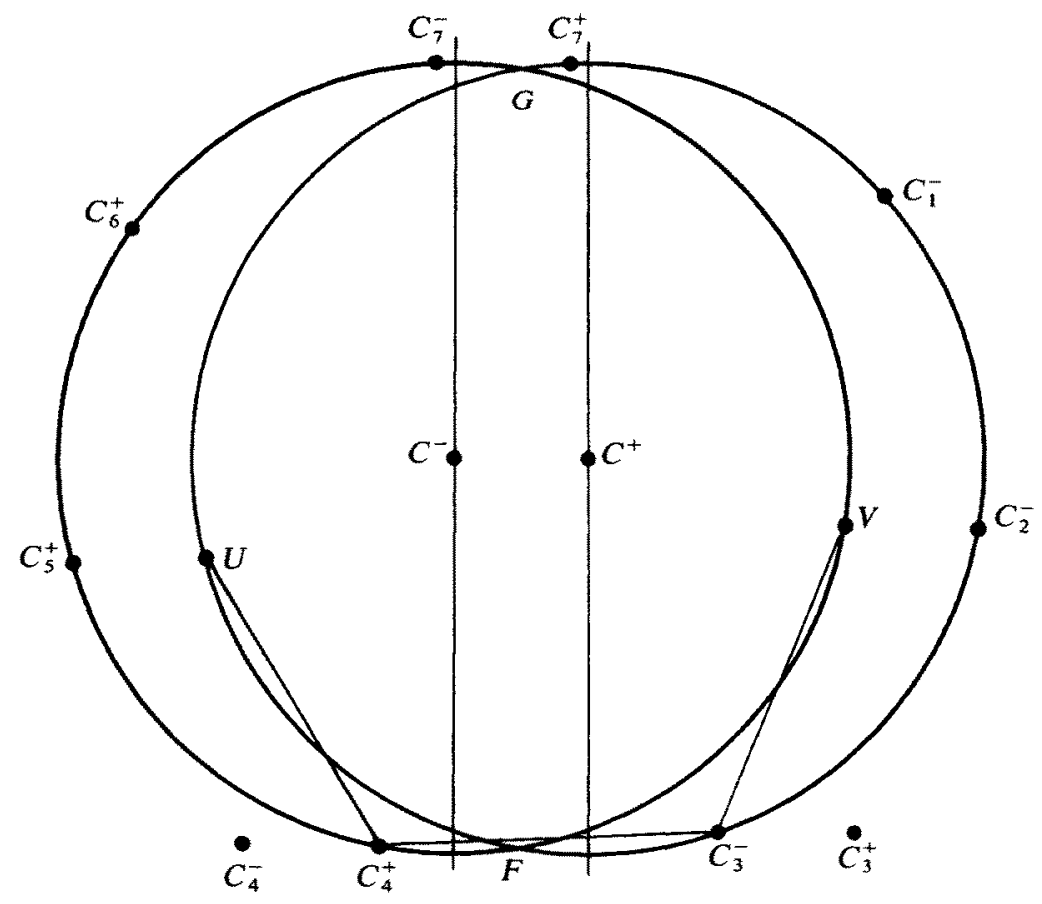

Fig. 2

this cyclic order, on bd( $\left.2 k^{-} \cup 2 k^{+}\right)$(Fig. 2). Translate $C_{5}^{+}$through the vector $\mathrm{C}^{-} \mathrm{C}^{+}$and $C_{2}^{-}$through $\mathrm{C}^{+} \mathrm{C}^{-}$obtaining the points $U$ and $V$. Let bd $2 k^{-}$and bd $2 k^{+}$intersect at the points $F$ and $G, G$ being in the same half-plane $y>0$ or $y<0$ as $C_{7}^{-}$and $C_{7}^{+}$. Let $\Sigma$ and $\sigma$ be the length of the arcs on bd $2 k^{+}$subtended by the chords of length 2 and $2 t$, respectively. Using an obvious notation for the length of an arc on bd $2 k^{-}$or bd $2 k^{+}$, we have

$$
\begin{aligned}
8 \pi / \sqrt{3}+2 \sigma & =\overparen{C_{7} G}+\widehat{G C_{7}^{+}}+\widehat{C_{7}^{+} C_{2}^{-}}+\widehat{C_{2}^{-} F}+\overparen{F C_{5}^{+}}+\overparen{C_{5}^{+} C_{7}^{-}} \\
& =\sigma+\widehat{C_{7}^{+} C_{2}^{-}}+\widehat{V F}+\sigma+\widehat{F U}+\sigma+\widehat{C_{5}^{+} C_{7}^{-}} \geq 4 \Sigma+3 \sigma+\widehat{U F}+\widehat{F V},
\end{aligned}
$$

whence

$$
\Lambda=\overparen{U F}+\widehat{F V}+\sigma+4 \Sigma-8 \pi / \sqrt{3} \leq 0 .
$$

For a given value of $t, \widehat{U F}+\widehat{F V}$ attains its minimum if, in the inequalities $C_{4}^{+} C_{3}^{-} \geq 2, C_{4}^{+} U=C_{4}^{-} C_{5}^{+} \geq 2, C_{3}^{-} V=C_{3}^{+} C_{2}^{-} \geq 2$, equality holds. Geometrical considerations suggest, and numerical computations, support the conjecture that in the extremal position either $C_{4}^{+}$or $C_{3}^{-}$coincides with $F$. From the computed values of $\Lambda$ belonging to different values of $t$ and different positions of $C_{4}^{+}$and $C_{3}^{-}$we present, for a few values of $t$, only the smallest one which belong to the 
suggested position:

\begin{tabular}{cccccccc}
\hline$t$ & 0 & 0.065 & 0.066 & 0.1 & 0.2 & 0.3 & 0.4 \\
$\Lambda$ & -0.0313 & -0.0002 & 0.0004 & 0.0156 & 0.0572 & 0.0944 & 0.1280 \\
$t$ & 0.5 & 0.6 & 0.7 & 0.8 & 0.9 & 1 & \\
$\Lambda$ & 0.1588 & 0.1954 & 0.2140 & 0.2390 & 0.2626 & 0.2852 & \\
\hline
\end{tabular}

This table shows that there is a value $\bar{t}=0.065 \cdots$ such that for $t>\bar{t}$ case (ii) cannot occur.

The same argument shows that case (ii) (for $t \leq \bar{t}$ ) cannot occur either if, from all seven pairs of centers, at least one center lies in $\lambda c^{-} \cup \lambda c^{+}$where $\lambda=1 / \sin (\pi / 7)$ is the circumradius of a regular heptagon of side-length 2 . But if for some $j \leq 7$ both $c_{j}^{-}$and $c_{j}^{+}$are outside $\lambda c^{-} \cup \lambda c^{+}$then $\left|s_{j}\right|$ is very small, more exactly less than $3|w|=0.00067 \cdots$ where $w$ is a circular segment cut off from $k^{+}$by a tangent of $(\lambda / 2) c^{+}$. On the other hand, $u_{j}$ prevents the rest of the centers under consideration from getting close to bd $2 k^{-} \cup 2 k^{+}$, so that inequality (2) is again amply fulfilled.

Case (iii) can be settled in a similar manner to (ii). Without going into details we mention that there is a constant $\bar{t}=0.034 \cdots$ such that for $t>\bar{t}$ case (iii) cannot occur.

This ends the proof of the theorem.

We still make some remarks about $d_{n}=\sup _{u \in S_{n}} d(u)$, where $S_{n}$ is the set of all possible unions of $n$ unit circles.

Let $u$ be the union of $n$ unit circles with centers on a line equally spaced at a distance $2 t \leq 2$. Then $\bar{d}(u)$ is given in terms of $t$ by the function

$$
f_{n}(t)=\frac{2(n-1)\left(\arcsin t+t \sqrt{1-t^{2}}\right)+\pi}{2(n-1) t \sqrt{4-t^{2}}+\sqrt{12}} .
$$

As we have seen, we have $d_{2}=\max _{0 \leq t \leq 1} f_{2}(t)$. Now we can phrase

Conjecture 2. For $n=3,4$, and 5 we have $d_{n}=\max _{0 \leq t \leq 1} f_{n}(t)$.

However, passing from $n=5$ to $n=6$ the extremal configuration seems to change drastically. Let $v$ be the union of six unit circles centered at the vertices of a regular hexagon of side-length 1 . Then we have

$$
\bar{d}(v)=\frac{3 \sqrt{3}+2 \pi}{(\sqrt{3} / 2)(7+3 \sqrt{5})}=0.96695 \ldots,
$$

whilst $\max _{0 \leq t \leq 1} f_{6}(t)=0.96686 \ldots$ This phenomenon is similar to the "sausage catastrophe" observed by Wills [6]. 
Improving a construction suggested by J. Pach we now shall show that

$$
\lim _{n \rightarrow \infty}\left(1-d_{n}\right) n \leq \frac{4 \sqrt{3}-2 \pi}{3 \sqrt{3}}
$$

Let $k>1$ and $l \geq 0$ be integers. In the thinnest lattice-covering of the plane with unit circles the centers of the circles form a lattice generated by the vertices of a regular triangle of side-length $\sqrt{3}$. Let $H$ be a closed regular hexagon of side-length $\sqrt{3} k$ containing $3 k(k+1)+1$ lattice-points. On bd $H$ we put, on each segment determined by consecutive lattice-points $l$, new points so as to divide the segment into $l+1$ parts of length $2 t=\sqrt{3} /(l+1)$. Now we have in $H$ altogether

$$
n=1+3 k(k+1)+6 k l
$$

points. Let $U$ be the union of the unit circles centered at these points. We have

$$
\begin{aligned}
|U| & =|\operatorname{conv} U|-|\operatorname{conv} U \backslash U| \\
& =\frac{9 \sqrt{3}}{2} k^{2}+6 \sqrt{3} k+\pi-6 k(l+1)\left(2 t-\arcsin t-t \sqrt{1-t^{2}}\right)
\end{aligned}
$$

where conv $U$ denotes the convex hull of $U$.

In the densest lattice-packing of translates of $U$ each domain is touched by six others so that adjacent domains have $2 k l-1$ points of contact. A unit cell $C$ of this lattice is given by joining to $H$ along three consecutive sides parallelograms of side-length 2 and $\sqrt{3} k$ including an angle $\arcsin \left(\frac{1}{2} \sqrt{4-t^{2}}\right)$ and two equilateral triangles of side-length 2 between the parallelograms. Thus we have

$$
|C|=\frac{9 \sqrt{3}}{2} k^{2}+3 \sqrt{3} k \sqrt{4-t^{2}}+2 \sqrt{3}
$$

and

$$
|C|-|U|=3 \sqrt{3} k\left(\sqrt{4-t^{2}}-\frac{1}{t} \arcsin t-\sqrt{1-t^{2}}\right)+2 \sqrt{3}-\pi
$$

Since

$$
\lim _{t \rightarrow 0} \frac{1}{t^{2}}\left(\sqrt{4-t^{2}}-\frac{1}{t} \arcsin t-\sqrt{1-t^{2}}\right)=\frac{1}{12}
$$

there is a positive constant $c$ such that for $0<t \leq \sqrt{3} / 2$ we have

$$
\sqrt{4-t^{2}}-\frac{1}{t} \arcsin t-\sqrt{1-t^{2}}<c t^{2}
$$


It follows that

$$
1-d_{n} \leq \frac{|C|-|U|}{|C|}<\frac{3 \sqrt{3} c k t^{2}+2 \sqrt{3}-\pi}{\frac{9 \sqrt{3}}{2} k^{2}}
$$

Let $p$ be a positive number less than $1 / 8$. Letting $n$ tend to infinity and choosing $l$ so that $n^{p}<l \leq m^{1 / 2-p}$ we have $\lim _{n \rightarrow \infty} n / k^{2}=3$ and $\lim _{n \rightarrow \infty} k / l^{2}=0$. Therefore multiplying the last inequality by $n$ and going over to the limiting value as $n \rightarrow \infty$ we obtain inequality (4).

It is conjectured that in (4) the sign of equality holds. We formulate the following sharper

Conjecture 3. Let $D(u)$ be the density of the densest packing of congruent copies of a domain $u$. Then

$$
\sup _{u \in S_{n}} D(u) \sim 1-\frac{4 \sqrt{3}-2 \pi}{3 \sqrt{3} n}
$$

\section{References}

1. C. A. Rogers, The closest packing of convex two-dimensional domains. Acta Math. 86 (1951), 309-321.

2. L. Fejes Tóth, Some packing and covering theorems. Acta Sci. Math. (Szeged) 12/A (1950), 62-67.

3. L. Fejes Tóth, On the densest packing of convex discs. Mathematika 30 (1983), 1-3.

4. L. Fejes Tóth, Densest packing of translates of a domain. Acta Math. Acad. Sci. Hungar. 45 (1984), 437-440.

5. L. Fejes Tóth, Über einen geometrischen Satz. Math. Z. 46 (1940), 83-85.

6. J. M. Wills, Research Problem 35. Period. Math. Hungar. 14 (1983), 312-314.

Received December 4, 1985. 